\title{
Accurate Calculation of CBED Patterns for 4D STEM Using Electron Densities Calculated by Density Functional Theory.
}

\author{
Mark P. Oxley ${ }^{1}$, Axiel Yaēl Birenbaum ${ }^{1}$, Tribhuwan Pandey ${ }^{1}$, Valentino R. Cooper ${ }^{1}$ and Miaofang Chi ${ }^{2}$ \\ 1. Oak Ridge National Laboratory, Materials Science and Technology Division, Oak Ridge TN, USA. \\ 2. Oak Ridge National Laboratory, Center for Nanophase Materials Sciences, Oak Ridge TN, USA.
}

The development of high-speed pixelated electron detectors has presented many opportunities for Scanning Transmission Electron Microscopy (STEM). The collection of Convergent-Beam Electron Diffraction (CBED) patterns as a function of probe position provides a feature rich data set with many possible means of analysis. These include the reconstruction of conventional imaging modes via the use of virtual apertures, exit wave function reconstruction using ptychography [1], and Differential Phase Contrast (DPC) imaging measuring local electric and magnetic fields [2].

In Fig. 1 we show examples of simulated CBED patterns for different probe positions in the $\mathrm{SrTiO}_{3}$ (STO) unit cell. These calculations were performed using the quantum excitations of phonons (QEP) option in the $\mu \mathrm{STEM}$ package [3]. The scattering potential here is constructed from scattering factors for isolated neutral atoms. While this is excellent for most image simulations, it has been recently shown that it does not correctly predict the electric field within STO and scattering factors for ions were used to simulate the measured beam deflection in DPC [2]. While this may be a viable approach for simple systems, for more complex structures such as interfaces and defects the choice of ionic scattering factors is not so straight forward. An alternative is to calculate the electron scattering factors based on electron densities determined from DFT. This approach has been shown to be necessary to simulate offaxis electron holography [4].

Electron scattering is primarily Coulomb scattering from the combined charge density of the electrons and the nuclear charge. The elastic electron scattering potential can be written as

$$
V(\mathbf{r})=\frac{1}{4 \pi \varepsilon_{0}}\left[\int \frac{\rho\left(\mathbf{r}^{\prime}\right)}{\left|\mathbf{r}-\mathbf{r}^{\prime}\right|} d \mathbf{r}^{\prime}-\sum_{n} \frac{Z_{n}}{\left|\mathbf{r}-\mathbf{R}_{n}\right|}\right]
$$

where $\rho$ is the sum of the single particle electron densities and $Z_{n}$ the atomic number of the nucleus located at position $\mathbf{R}_{n}$. In practice the integration of the electron density is arduous due to the long-range nature of the Coulomb potential, and instead the electron scattering factors are calculated by Fourier transforming the electron charge densities to obtain the $\mathrm{x}$-ray scattering factors and the nuclear charge is added using the Bethe-Mott formula.

Many DFT codes use pseudopotentials and calculate only the slowly varying electron density due to valance electrons. Previous authors have commonly applied correction factors to the resulting scattering potential by calculating potentials for isolated atoms for both pseudopotentials and all-electron codes [46]. We will use VASP, which can also output the frozen-core electron densities, to construct our model scattering potentials. These will be compared with scattering potentials calculated from all-electron densities from the Wien2K package.

QEP/frozen phonon calculations are carried out using multiple passes through different atomic configurations to model the motion of the nucleus. This typically involves moving the entire atomic 
scattering potential. It would be impractical to do DFT calculations for many different configurations and we will shift only the nuclear and core electron potentials. As can be seen from Fig. 2, these are for more localized than the scattering potential calculated for the valence electrons.

We will discuss practical schemes to allow the routine simulation of 4D STEM data using realistic scattering potentials. The correct scattering potential is only a starting point for quantitative analysis of CBED patterns. We will also discuss the effects of temporal and spatial incoherence on the CBED intensities. While simple Einstein models suffice to describe atomic motions for most imaging modes, detailed descriptions of phonon dispersions may also be required. Finally, the importance of probe aberrations will be discussed.

\section{References:}

[1] H. Yang et al., Nature Communications 7 (2016) p. 12532.

[2] N. Shibata et al, Nature Communications 8 (2017) 15631.

[3] L.J. Allen, A.J. D’Alfonso and S.D. Findlay, Ultramicroscopy 151 (2015) p.11.

[4] S. Borghardt et al., Physical Review Letters 118 (2017) 086101

[5] W.L. Wang and E Kaxiras, Physical Revie B 87 (2013) 085103.

[6] L.C. Meyer et al., Nature Materials 10 (2011) p. 209.

[7] This research was supported by the US Department of Energy, Office of Basic Energy Sciences, Materials Sciences and Engineering Division, and ORNL's Center for Nanophase Materials Sciences, which is a U.S. DOE Office of Science User Facility.
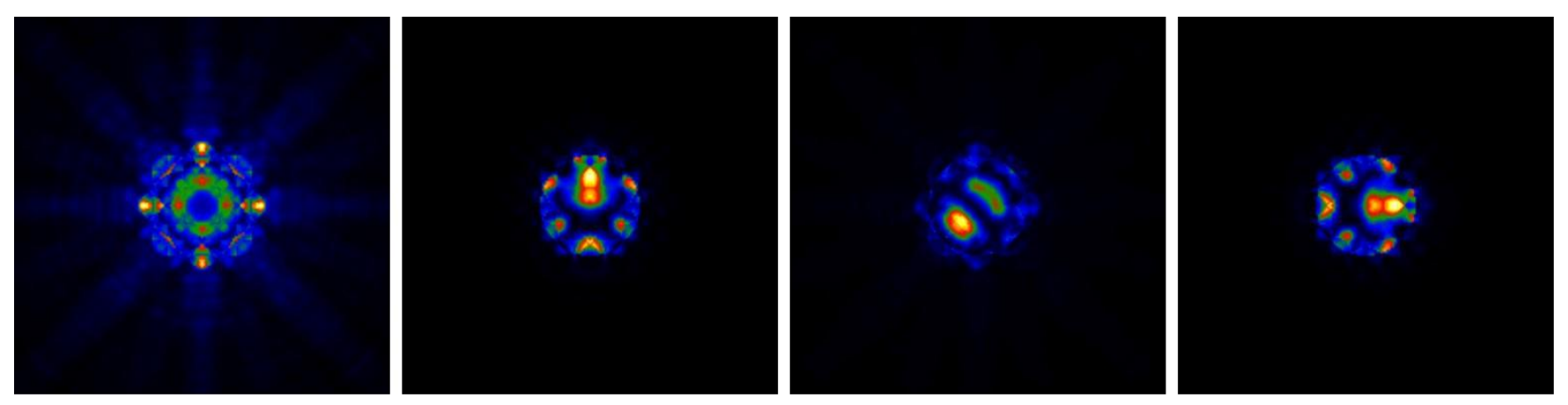

Figure 1. Simulated CBED patterns at various probe positions for a $100 \mathrm{kV}$ electron beam with a 30 mrad. probe forming aperture incident on $100 \AA$ thick $\mathrm{SrTiO}_{3}$.
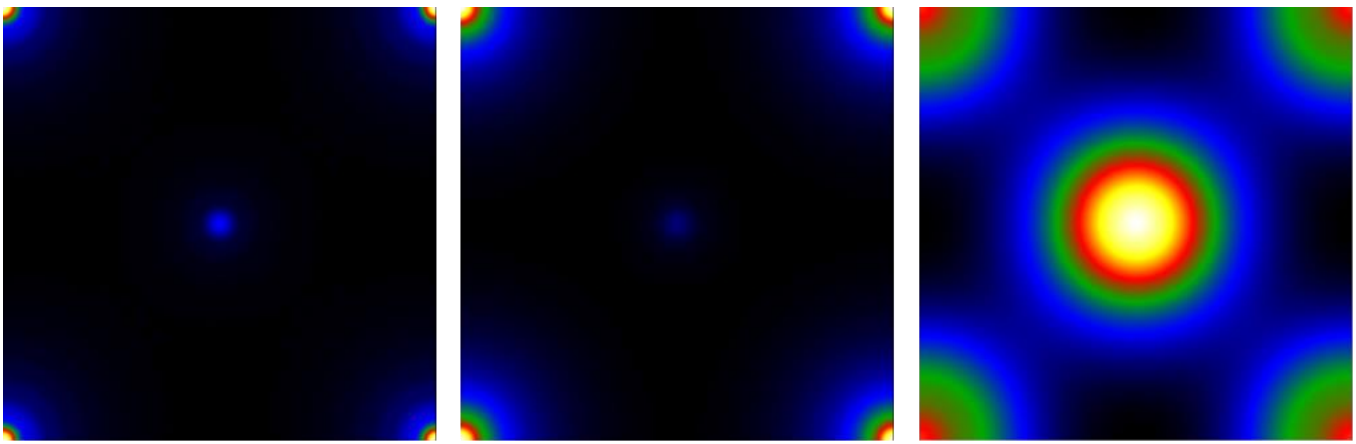

Figure 2. A slice of the 3-dimensional scattering potentials from the top of the $\mathrm{SrTiO}_{3}$ unit cell showing the nuclear potential (left), core electron potential (center) and valence electron potential (right). 